\title{
Pregnancy and cesarean delivery after multimodal therapy for vulvar carcinoma: A case report
}

\author{
KUNIAKI TORIYABE ${ }^{1,2}$, HARUKI TANIGUCHI ${ }^{2}$, TOKIHIRO SENDA ${ }^{2,3}$, \\ MASAKO NAKANO ${ }^{2}$, YOSHINARI KOBAYASHI ${ }^{2}$, MIHO IZAWA $^{2}$, HIROHIKO TANAKA $^{2}$, \\ TETSUO ASAKURA ${ }^{2}$, TSUTOMU TABATA ${ }^{1}$ and TOMOAKI IKEDA ${ }^{1}$ \\ ${ }^{1}$ Department of Obstetrics and Gynecology, Mie University Graduate School of Medicine, Tsu, Mie 514-8507; \\ ${ }^{2}$ Department of Obstetrics and Gynecology, Mie Prefectural General Medical Center, Yokkaichi, Mie 510-8561; \\ ${ }^{3}$ Department of Obstetrics and Gynecology, Kinan Hospital, Mihama, Mie 519-5293, Japan
}

Received November 4, 2015; Accepted September 12, 2016

DOI: $10.3892 / \mathrm{mco} .2016 .1021$

\begin{abstract}
Reports of pregnancy following treatment for vulvar carcinoma are extremely uncommon, as the main problem of subsequent pregnancy is vulvar scarring following radical surgery. We herein report the case of a patient who was diagnosed with stage I squamous cell carcinoma of the vulva at the age of 17 years and was treated with multimodal therapy, including neoadjuvant chemotherapy, wide local excision with bilateral inguinal lymph node dissection and adjuvant radiotherapy. The patient became pregnant spontaneously 9 years after her initial diagnosis and the antenatal course was good, except for mild acute pyelonephritis at 25 weeks of gestation. An elective caesarean section was performed at 38 weeks of gestation due to vulvar scarring following multimodal therapy, particularly radiotherapy. The patient remains alive and well, without signs of recurrence or metastasis 12 years after her diagnosis and treatment. Radical vulvectomy as well as multimodal therapy for vulvar carcinoma, particularly radiotherapy, may cause extensive skin scarring. The presence of vulvar scarring following multimodal therapy for vulvar carcinoma may increase the incidence of caesarean delivery.
\end{abstract}

\section{Introduction}

As carcinoma of the vulva is rare among young women, reports of pregnancy following treatment for vulvar carcinoma are extremely uncommon. The incidence of vulvar carcinoma, however, has recently increased among younger women; thus, there may have been an increase in pregnancy rates following vulvar carcinoma treatment (1). Pregnant women who undergo surgical treatment for vulvar carcinoma, including radical

Correspondence to: Dr Kuniaki Toriyabe, Department of Obstetrics and Gynecology, Mie University Graduate School of Medicine, 2-174 Edobashi, Tsu, Mie 514-8507, Japan

E-mail: to.kuniaki@gmail.com

Key words: vulvar carcinoma, pregnancy, multimodal therapy, squamous cell carcinoma, caesarean delivery vulvectomy, may have an increased incidence of caesarean delivery (2). In the literature, vulvar scarring following radical vulvectomy was the major reason for pregnant women undergoing caesarean section (2-7). To date, no cases of pregnancy following vulvar carcinoma have been reported in patients who had undergone surgery and radiotherapy.

We herein describe a case in which caesarean section was performed due to the presence of extensive vulvar scarring following multimodal therapy for vulvar carcinoma, including chemotherapy, surgery and radiotherapy.

\section{Case report}

In April, 2003, a 17-year-old, nulligravida Japanese woman, who was a smoker, presented with a 4-month history of a mass (sized $>2 \mathrm{~cm}$ ) with accompanying pruritus in the left labium majus (Fig. 1). Examination of biopsy specimens revealed invasive squamous cell carcinoma with keratin pearl formation and stromal invasion $>1 \mathrm{~mm}$ in depth (Fig. 2). Screening for sexually transmitted infections, including HIV serology, syphilis serology, Chlamydia trachomatis polymerase chain reaction and Neisseria gonorrhoeae polymerase chain reaction were performed, and were all negative. The serum squamous cell carcinoma antigen level was $2.6 \mathrm{ng} / \mathrm{ml}$ (normal value, $<1.5 \mathrm{ng} / \mathrm{ml}$ ). Magnetic resonance imaging (MRI) revealed enlarged inguinal lymph nodes bilaterally. The patient first underwent neoadjuvant chemotherapy (pepleomycin, $5 \mathrm{mg} /$ body intramuscular injection twice a week for 2.5 weeks, 5 cycles of administration in total). The tumor of the vulva was reduced in size following chemotherapy. Subsequently, wide local excision with bilateral inguinal lymph node dissection was performed (Figs. 3 and 4). All surgical margins $(\geq 1 \mathrm{~cm})$ were free of carcinoma cells. The lymph nodes were negative for malignancy and there was no evidence of lymphovascular invasion. The tumor was classified as ypT1bNOM0, and a diagnosis of International Federation of Gynecology and Obstetrics (2008) stage IB keratinizing squamous cell carcinoma of the vulva was confirmed. The specimen was negative for p16 immunohistostaining. The skin surrounding the cancer lesion displayed non-specific chronic eczema, without any vulvar intraepithelial neoplasia. One month after the vulvar 


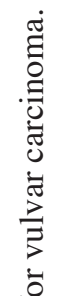

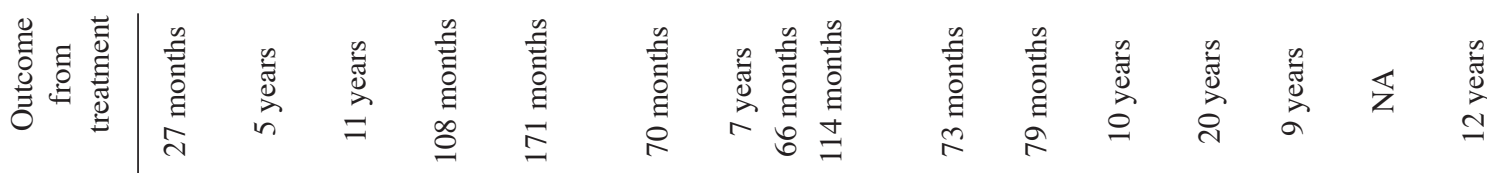

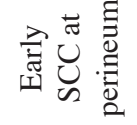

䘮㠩艺

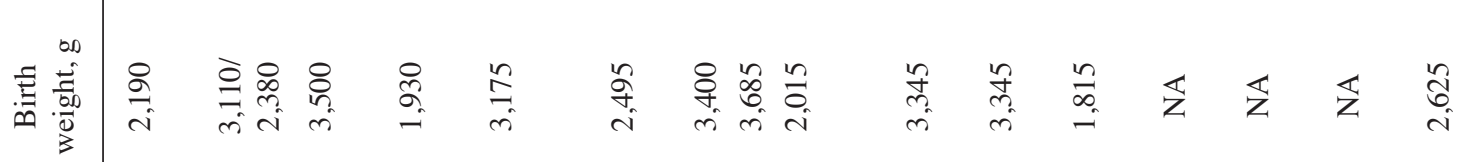

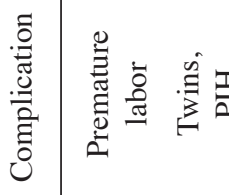

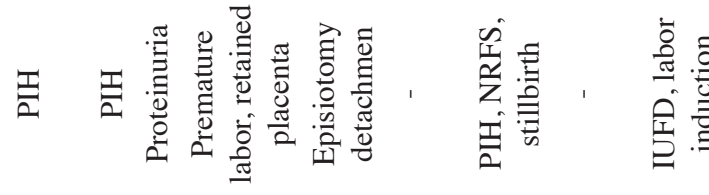

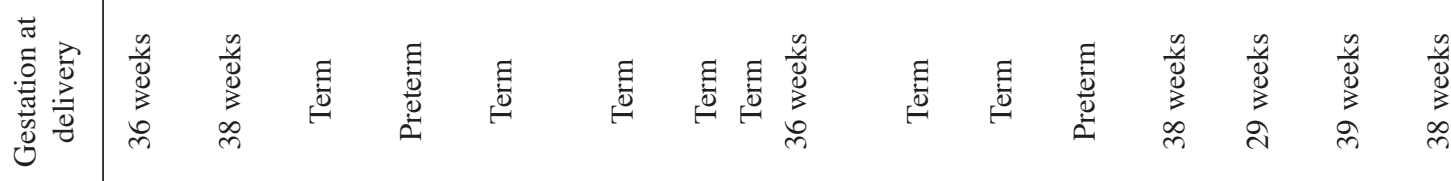

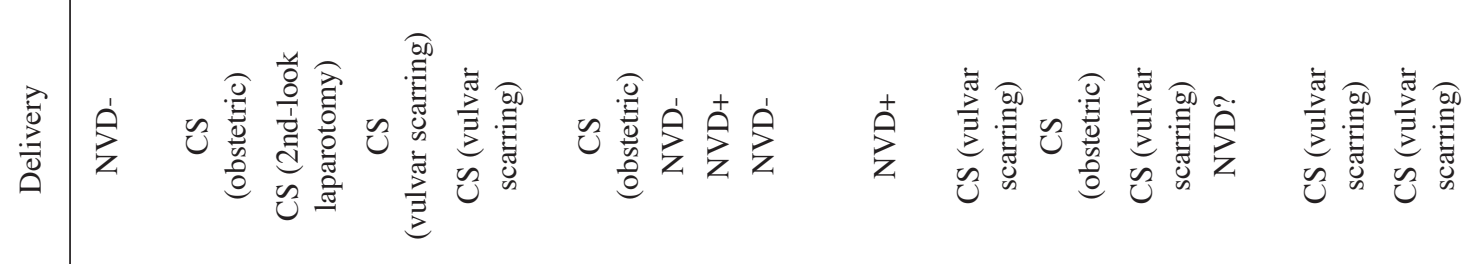
节

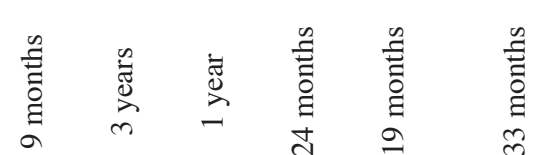

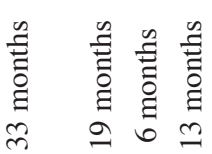

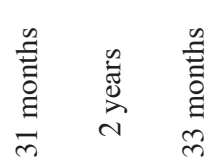

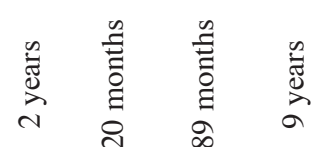

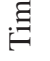

惫密

竞蜜

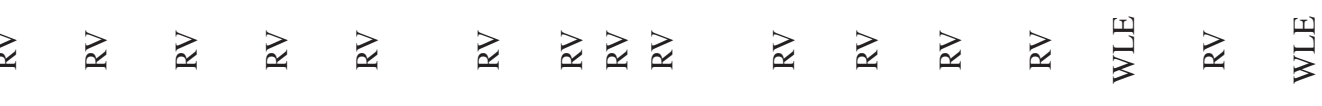

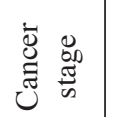
$\geq$

高

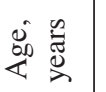

n

n

2

$\stackrel{\infty}{\sim}$

일

กี

$m$ ले

ते ஜ గ =

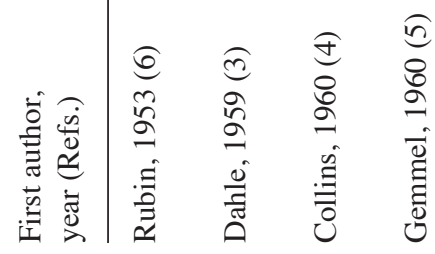

童

高㩆

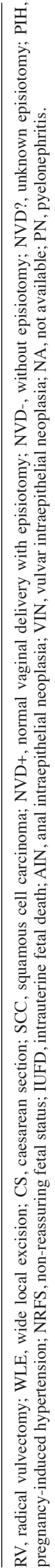




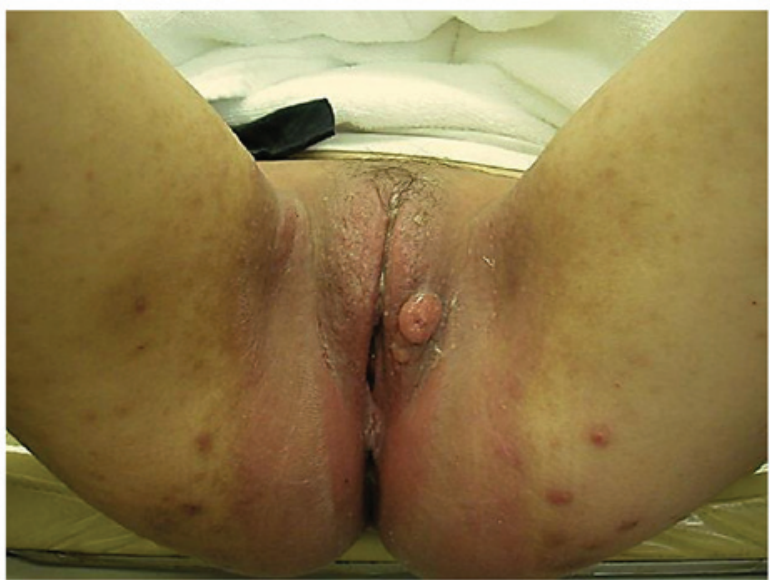

Figure 1. A tumor sized $>2 \mathrm{~cm}$ was identified on the left labium majus on physical examination.

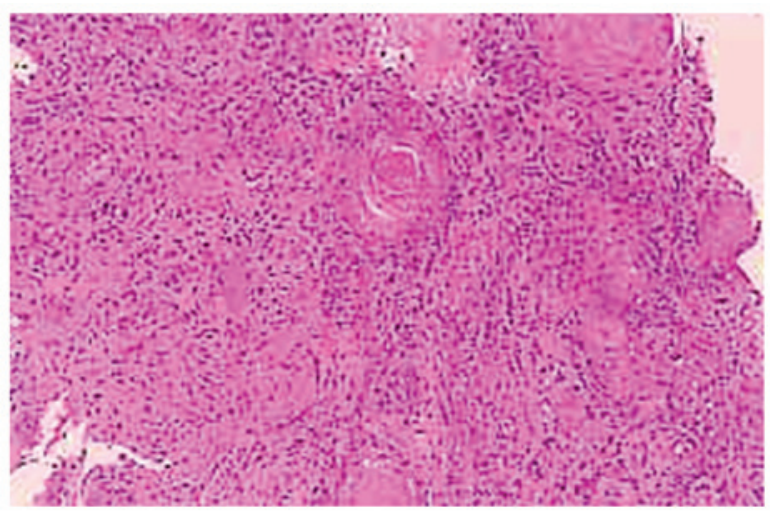

Figure 2. Biopsy specimen of the vulvar tumor showing invasive squamous cell carcinoma with keratin pearl formation, with stromal invasion of $>1 \mathrm{~mm}$ in depth (magnification, $\mathrm{x} 40$ ).

surgery, the patient received adjuvant radiotherapy of the vulvar and inguinal regions bilaterally (external-beam irradiation, $1.8 \mathrm{~Gy} /$ day in 25 fractions over 5 weeks; total radiation dose, $45.0 \mathrm{~Gy})$.

The patient was prescribed an ointment of steroids and zinc oxide for radiation dermatitis of the vulva and perineum by the dermatologist. There was no dysuria or dyschezia following radiotherapy. No stenosis of the vaginal orifice occurred, but the patient occasionally experienced mild dyspareunia, for which she used lubricants during coitus. The menstrual cycles following multimodal therapy were regular.

The patient became pregnant spontaneously in January, 2012, at the age of 26 years, 9 years after the diagnosis and treatment of her malignancy. Delivery by caesarean section was planned, due to vulvar scarring extending to the perineum and anal granulation following multimodal therapy, particularly radiotherapy (Fig. 5). There was no edema on the vulva or the lower limbs during pregnancy. The antenatal course was good, except for mild acute pyelonephritis at 25 weeks of gestation, which was considered to be unrelated to her previous treatment. The patient improved after 3 days of intravenous administration of cefmetazole, and there was no relapse of pyelonephritis until delivery. In August, 2012, a healthy female infant was delivered by elective caesarean

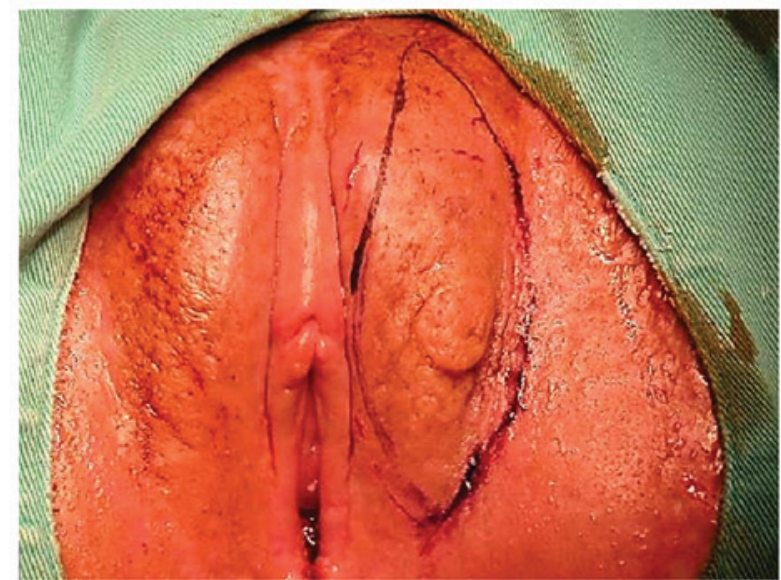

Figure 3. The vulvar tumor was reduced in size following neoadjuvant chemotherapy and wide local excision was planned.

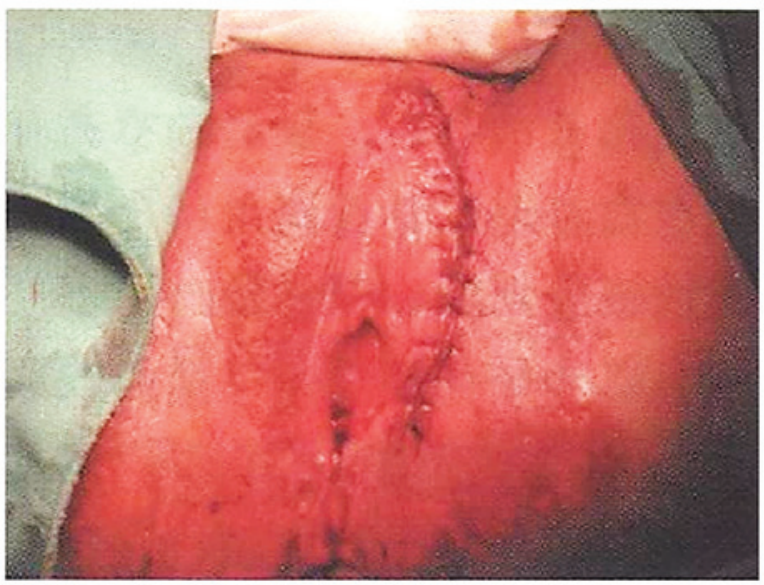

Figure 4. The vulvar tumor was resected with a surgical margin of $\geq 1 \mathrm{~cm}$ and the wound was closed using the knotted suture technique.

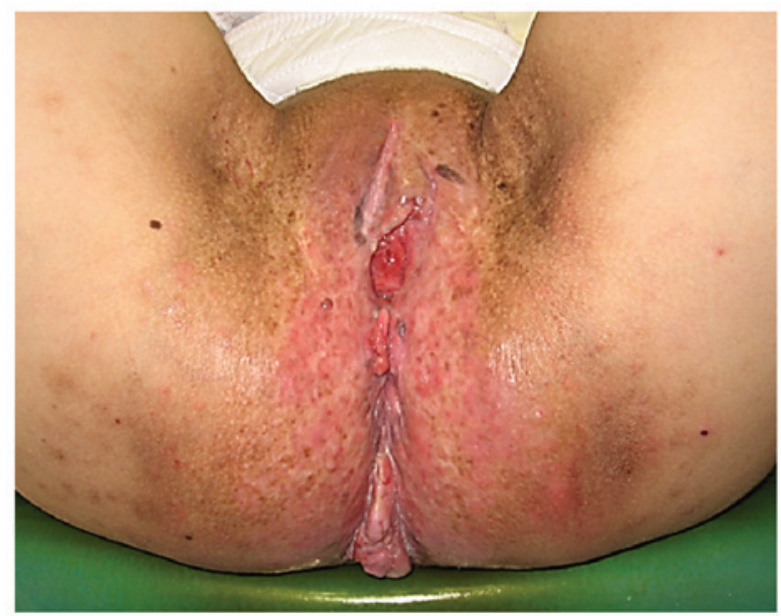

Figure 5. Vulvar scarring extending to the perineum and anal granulation.

section under combined spinal epidural anesthesia, at a gestational age of 38 weeks and 2 days. The amount of intraoperative hemorrhage was $1,926 \mathrm{ml}$, containing amniotic fluid. The newborn weighed 2,625 g and was $45.0 \mathrm{~cm}$ tall, with an Apgar 
score of 8 both at 1 and $5 \mathrm{~min}$. The umbilical cord arterial $\mathrm{pH}$ was 7.330 and base excess was -3.1. Placental pathology revealed no abnormal findings.

In April, 2015 (12 years after the initial diagnosis of vulvar carcinoma), the patient was alive and well, without signs of recurrence or metastasis. At the last follow-up, the child was aged 2 years and exhibited good growth and development, except for a case of lymphadenitis purulenta of the neck with febrile convulsions at the age of 1 year.

\section{Discussion}

Reports of pregnancy following treatment for vulvar carcinoma are extremely uncommon. Our case involved a Japanese woman who became pregnant after having been treated for vulvar carcinoma. We reviewed 15 cases reported in the literature (Table I) and all the patients remained alive (2-7). Only 1 patient had stage IV disease (metastasis to the pelvic lymph nodes), whereas the others had stage I disease. Vulvar surgery involved 1 case undergoing wide local excision and 14 cases of radical vulvectomy. Radiation therapy was not administered in any of the cases. Recurrence of vulvar lesions was confirmed in 3 of the 14 cases with a long follow-up reported in the literature. All 3 recurrent cases were treated by local excision. Of the 10 cases who were nulliparous at treatment for vulvar cancer, 8 underwent caesarean section; the remaining 2 cases had a normal vaginal delivery (premature labor in 1 case and labor induction for intrauterine fetal death in the other case). Of the 5 parous cases at treatment for malignancy, 4 had a normal vaginal delivery and the remaining patient underwent a caesarean section. Thus, the mode of delivery following treatment for vulvar carcinoma may be affected by parity at treatment.

The major reason for caesarean section being performed in the reported cases was vulvar scarring following treatment for vulvar carcinoma; however, the obstetrical reasons were minor. In our review, 5 of 9 cases who had undergone caesarean delivery had vulvar scarring following radical vulvectomy for vulvar carcinoma. In contrast to the reported cases, however, the vulva of our patient was scarred following multimodal therapy for vulvar cancer, including chemotherapy, surgery and radiotherapy. For vulvar carcinoma, surgical therapy, including wide local excision, simple vulvectomy and radical vulvectomy, with or without bilateral inguinal lymph node dissection, are currently the standard treatment. In certain cases, however, radiotherapy with or without chemotherapy may also be performed in addition to surgical therapy $(8,9)$.
Our patient first presented with a vulvar tumor sized $>2 \mathrm{~cm}$ and enlarged inguinal lymph nodes on MRI at the age of 17 years. Following tumor shrinkage with neoadjuvant chemotherapy, wide local excision was performed. Subsequently, although the inguinal lymph nodes were negative for malignancy, adjuvant radiotherapy was administered for potential future local recurrence and inguinal lymph node metastasis. Although our case did not undergo major surgery on the vulva, radiotherapy caused extensive skin scarring, extending from the vulva to the perineum. Moreover, granulation tissue developed around the anus. Thus, an elective caesarean delivery was planned to avoid complicated lacerations during delivery.

In conclusion, pregnancy following treatment for vulvar carcinoma may be accompanied by post-treatment vulvar scarring. In addition to radical vulvectomy, multimodal therapy, particularly radiotherapy, may also cause extensive skin scarring. When considering radiotherapy for vulvar carcinoma in young patients, the risk of vulvar scarring and the probability of future delivery by caesarean section should be explained to the patients.

\section{References}

1. Hampl M, Deckers-Figiel S, Hampl JA, Rein D and Bender HG: New aspects of vulvar cancer: Changes in localization and age of onset. Gynecol Oncol 109: 340-345, 2008.

2. Palmer JE and Tidy JA: Pregnancy following vulvar squamous cell carcinoma: A report of two cases. J Gynecol Oncol 20: 254-256, 2009.

3. Dahle T: Carcinoma of the vulva and subsequent successful pregnancy. Acta Obstet Gynecol Scand 38: 448-452, 1959.

4. Collins JH, Birch HW, Pailet M and Avent JK: Pregnancy and delivery following extensive vulvectomy. Am J Obstet Gynecol 80: 167-171, 1960.

5. Gemmell AA and Haines M: Pregnancy following radical vulvectomy for carcinoma of the vulva. J Obstet Gynaecol Br Emp 67: 199-207, 1960.

6. Rubin A and Lewis GC Jr: Pregnancy and vaginal delivery following radical surgery for cancer of the vulva; review of the literature and case report. Am J Obstet Gynecol 65: 1347-1349, 1953.

7. Arjona JE, Velasco E, Cervelo P, Espejo E, Pizarro I, Carrasco S and Castelo-Branco C: Pregnancy following radical vulvectomy for carcinoma of the vulva: A case report and literature review. Eur J Obstet Gynecol Reprod Biol 158: 113-114, 2011.

8. Mahner S, Jueckstock J, Hilpert F, Neuser P, Harter P, de Gregorio N, Hasenburg A, Sehouli J, Habermann A, Hillemanns P, et al; AGO-CaRE 1 investigators: Adjuvant therapy in lymph node-positive vulvar cancer: The AGO-CaRE-1 Study. J Natl Cancer Ins 107: dju426, 2015.

9. Matsuo K, Whitman SA, Blake EA, Conturie CL, Ciccone MA, Jung CE, Takiuchi T and Nishimura M: Feto-maternal outcome of pregnancy complicated by vulvar cancer: A systematic review of literature. Eur J Obstet Gyn Reprod Biol 179: 216-223, 2014. 